\title{
Nanoparticles of Low Optical Band Gap Conjugated Polymers
}

\author{
Johannes Huber, Christoph Jung, and Stefan Mecking* \\ Chair of Chemical Materials Science, Department of Chemistry, University of Konstanz, Universitätstrasse 10, D-78457 Konstanz, \\ Germany
}

\begin{abstract}
Sonogashira coupling of 9,9-bis (2-ethylhexyl)2,7-diethynyl-9H-fluorene (1) with 2,7-dibromo-9,9-bis (2ethylhexyl)-9H-fluorene (2) and dibromo-substituted electron accepting arylenes 5,7-bis (5-bromothiophen-2-yl)-2,3dihexylthieno $[3,4-6]$ pyrazine (3), 4,7-bis (5-bromo-4-(2ethylhexyl)thiophene-2-yl) bis(benzothiadiazole) (4) or 4,9bis (5-bromo-4-(2-ethylhexyl) thiophen-2-yl)-6,7. dihexylthiadiazolo[ $3,4-g]$ quinoxaline $(5)$, respectively, in a miniemulsion polymerization process afforded colloidally stable dispersions of poly (arylene ethynylene) nanoparticles

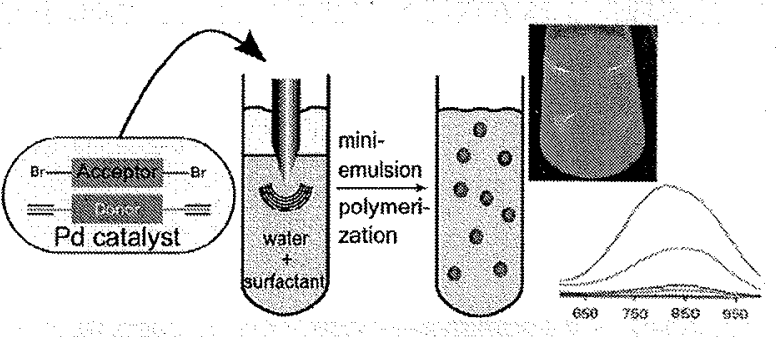
with an average size in the range of 50 to $120 \mathrm{~mm}$. For these poly $[(1-$ alt $-(2-\operatorname{co}-\mathrm{X})](\mathrm{X}=3,4$, or 5$)$, poly $(1-$ alt-3), poly $(1-$ alt-4), and poly $(1-a l t-5)$ aqueous nanoparticle dispersions absorption occurs increasingly in the red and NIR regime with increasing incorporation of acceptor monomer, with a strong absorption up to $\lambda_{\mathrm{abs}}=1 \mu \mathrm{m}$ for poly $(1$-alt-4) dispersions. For poly $[1$-alt- $(2-$ co-3) ] nanoparticles fluorescence is also observed at $\lambda_{\mathrm{em}}=\mathrm{ca} .700 \mathrm{~nm}$, which occurs at this long wavelength exclusively even at an incorporation of only $1 \mathrm{~mol} \%$ of 3 due to energy transfer to the low-energy chromophores. An alternative postpolymerization approach by change of solvent quality yielded dilute aqueous dispersions of self-stabilized nanoparticles from an amphiphilic block copolymer, poly(ethylene glycol)-block-poly (1-alt-6)-block-poly(ethylene glycol) generated from alkyne-terminated poly $(1$-alt-6) via azide-alkyne coupling $(6=4,7$-bis ( 5 -bromo-4 (2-ethylhexyl) thiophene-2-yl)benzothiadiazole), which fluoresce at $\lambda_{\mathrm{em} \text { max }}=672 \mathrm{~nm}$.
\end{abstract}

\section{INTRODUCTION}

Nanoparticles of conjugated polymers are finding increasing attention due to their luminescent nature. ${ }^{1}$ They are studied for optoelectronics, $^{2,3}$ live biological imaging, ${ }^{4-6}$ or sensors. ${ }^{7-9}$

By comparison to other types of nanoparticles, conjugated polymer nanoparticles are characterized by high absorption coefficients, nonlinear optical absorption, and photostability. The systems studied to date largely absorb and emit at wavelengths below $600 \mathrm{~nm}$. However, there is a clear general need to cover also the red and near-infrared red (NIR) part of the spectrum. ${ }^{10-15}$ For example, this is desirable for excitation with less damaging, deeper penetrating light, multicolor imaging, NIR photothermal therapy or more efficient energy harvesting. To this end, nanoparticles of conjugated polymers containing small amounts of physically blended NIR-emitting low-molecular-weight organic dyes ${ }^{16}$ or inorganic quantum $\operatorname{dots}^{17}$ have been reported. Notably, bulk materials and thin films of conjugated polymers with low optical band gaps corresponding to absorption of high wavelength visible light are studied intensely in the context of organic solar cells. ${ }^{18,19}$ The lack of corresponding nanoparticles is likely related to the low solubility of such polymers and chromophors with condensed and multiple heterocycles. This is an issue in both direct nanoparticles synthesis by polymerization in disperse heterophase systems, ${ }^{20,21}$ as well as postpolymerization approaches. ${ }^{2,22-24}$ We now give a first full account of the preparation and some basic physical properties of nanoparticles of low optical band gap polymers.

\section{EXPERIMENTAL SECTION}

General Methods and Materials. NMR spectra were recorded on a Bruker Avance III 400 spectrometer. ${ }^{1} \mathrm{H}$ and ${ }^{13} \mathrm{C}$ resonances were referenced to the solvent signal. TEM images were obtained on a Zeiss Libra 120 instrument (acceleration voltage $120 \mathrm{kV}$ ). Samples were not contrasted. Dispersions were dialyzed for TEM analysis to remove any free surfactant, and applied to a carbon coated copper grid in diluted form. FT-IR spectra were recorded on a Perkin-Elmer Spectrum 100 spectrometer. Absorption spectra were recorded on a Varian Cary 50 spectrometer. Fluorescence spectra and fluorescence quantum yields were obtained on a Hamamatsu absolute PL quantum yield measurement system C9920-02, or with a custom-built NIR fluorescence spectrometer with a nitrogen-cooled Ge diode (Northcoast) as a detector. A diode laser (690 nm, $19 \mathrm{~mW}$, model ACM19/ 1203) was used for excitation. Miniemulsions were prepared using a Bandelin HD 2200 sonotrode with a KE76 tip operated at $120 \mathrm{~W}$. Gel permeation chromatography (GPC) was carried out on a Polymer Laboratories PL-GPC 50 instrument equipped with a refractive index and a UV/vis detector, with two PLgel $5 \mu \mathrm{m}$ MIXED-C columns in THF at $40{ }^{\circ} \mathrm{C}$ against polystyrene standards. Particle sizes were determined by dynamic light scattering (DLS) on a Malvern Nano-ZS ZEN 3600 particle sizer $\left(173^{\circ}\right.$ back scattering). The autocorrelation 


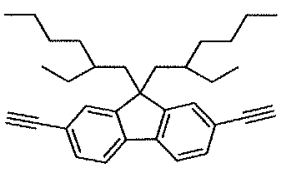

1

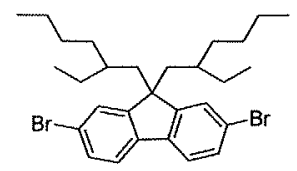

2

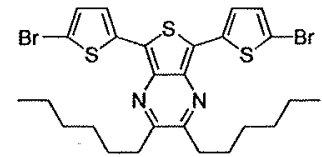

3

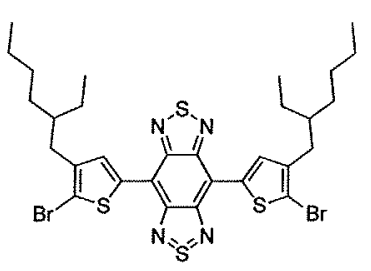

4

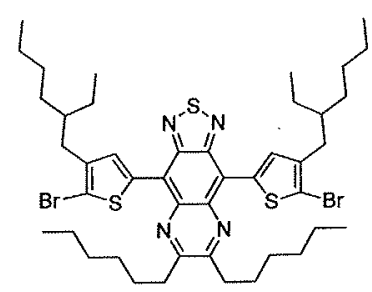

5

Figure 1. Monomers employed in miniemulsion polymerization to afford low band gap conjugated polymer particles.

function was analyzed using the Malvern dispersion technology software 5.1 algorithm to obtain volume weighted particle size distributions, and polydispersities.

Sodium dodecyl sulfate (SDS) (>99\%) (Fisher Scientific), [Pd$\left.\left(\mathrm{PPh}_{3}\right)_{4}\right](>99 \%)$ (MCAT), [Ni(dppp) Cl $]$ (Aldrich), CuI (98\%) (Aldrich), and $\mathrm{CuBr}(\geq 97 \%)$ (Merck) were used as received. Diisopropyl amine (>99.5\%) (Aldrich) was dried and distilled over $\mathrm{KOH}$, toluene was distilled from sodium, methanol was dried and distilled from magnesium, THF was distilled from sodium with benzophenone as a moisture indicator, and chloroform was dried over $\mathrm{CaH}_{2}$. All reactions were carried out under an argon atmosphere with standard Schlenk techniques unless stated otherwise. Azide-monofunctionalized poly(ethylene glycol) ( $\mathrm{PEG}_{2000^{-a z i d e}}$ $\left(\mathrm{CH}_{3}\left\{\mathrm{OCH}_{2} \mathrm{CH}_{2}\right\}_{n} \mathrm{~N}_{3}\right)_{\text {, }}^{25}$ and $\left[\mathrm{Pd}\left(\mathrm{PtBu}_{3}\right)_{2}\right]^{26}$ were prepared according to published procedures.

Synthesis of Monomers. 9,9-Bis(2-ethylhexyl)-2,7-diethynyl-9Hfluorene (1) and 2,7-dibromo-9,9-bis(2-ethylhexyl)-9H-fluorene (2) were synthesized as reported previously. ${ }^{5,27} 5,7-\mathrm{Bis}$ (5-bromothiophen2 -yl)-2,3-dihexylthieno[3,4-b] pyrazine ${ }^{28-30}(3)$ and 4,7-bis(5-bromo4-(2-ethylhexyl)thiophene-2-yl)bis(benzothiadiazole) $)^{32-34}$ (4) were prepared according to slightly altered literature protocols. 4,9-Bis ( 5 bromo-4-(2-ethylhexyl)thiophen-2-yl)-6,7-dihexylthiadiazolo[3,4-g]quinoxaline $\mathrm{e}^{28,3 \mathrm{I}-34}(\mathbf{5})$ was obtained by reported procedures. $4,7-$ Bis(5-bromo-4-(2-ethylhexyl)thiophene-2-yl)benzothiadiazole) (6) was generated in five steps from benzothiadiazole. ${ }^{35-37}$ (cf. the Supporting Information for experimental details of all monomer syntheses).

General Miniemulsion Polymerization Procedure. Stoichiometric amounts of the respective monomers were mixed with 80-120 $\mu \mathrm{g}$ of Cul, $1.0 \mathrm{~g}$ of toluene, $0.5 \mathrm{~g}$ of diisopropyl amine and $1 \mathrm{~mol} \%$ (relative to the total amount of monomers) $\left[\mathrm{Pd}\left(\mathrm{PPh}_{3}\right)_{4}\right]$ in a drybox The solution was drawn into a $5 \mathrm{~mL}$ syringe, transferred from the drybox, and injected into $25 \mathrm{~mL}$ of degassed 1 or $2 \mathrm{wt} \%$ (relative to water) SDS aqueous solution under an argon atmosphere in a $100 \mathrm{~mL}$ round-bottom flask fitted with a Teflon stopcock and a magnetic stirrer. The mixture was subjected to 2 min ultrasound treatment with a sonotrode under argon counterflow. The flask was sealed and heated to $50^{\circ} \mathrm{C}$ while stirring for several days. For further analysis, an aliquot of the dispersion was precipitated by addition to excess methanol, to afford a colorless cleat supernatant and the polymer in quantitative yield. Toluene and amine base residues can be removed from the dispersions by dialysis against neat water if necessary.

Block Copolymer Synthesis. A $223.4 \mathrm{mg}(0.327 \mathrm{mmol}, 0.818$ equiv) sample of 4,7-bis(5-bromo-4-(2-ethylhexyl)thiophene-2-yl)benzothiadiazole) (6) and $175.5 \mathrm{mg}(0.400 \mathrm{mmol}, 1$ equiv) of 9,9 . bis(2-ethylhexyl)-2,7-diethynyl-9H-fluorene (1) were degassed thoroughly, and dissolved in a mixture of $7 \mathrm{~mL}$ of dry toluene and $1 \mathrm{~mL}$ of piperidine. $\left[\mathrm{Pd}\left(\mathrm{PPh}_{3}\right)_{4}\right](4 \mathrm{mg})$ and $10 \mu \mathrm{g}$ of $\mathrm{CuI}$ were added to the mixture in a Schlenk tube. The reaction mixture was stirred at $60^{\circ} \mathrm{C}$ for $67 \mathrm{~h}$ and the polymer was then precipitated from methanol, filtered, washed with methanol and dried in vacuo. Molecular weights were determined by GPC and ${ }^{1} \mathrm{H}$ NMR analysis by integration of the ethynyl protons against the backbone protons (cf. Supporting Information).

A $20 \mathrm{mg}$ (1.6 $\mu \mathrm{mol}$ ethynyl end-groups) sample of the aforementioned alkyne-terminated poly(1-alt-6), $3.47 \mathrm{mg}(1.7 \mu \mathrm{mol})$ of $\mathrm{PEG}_{2000}$-azide $\left(\mathrm{CH}_{3}\left\{\mathrm{OCH}_{2} \mathrm{CH}_{2}\right\}_{n} \mathrm{~N}_{3}\right)$ and $2 \mathrm{mg}(13.9 \mu \mathrm{mol})$ of $\mathrm{CuBr}$ were dissolved in $1 \mathrm{~mL}$ dry THF under an argon atmosphere. The mixture was heated to $50^{\circ} \mathrm{C}$ for $94 \mathrm{~h}$. The sample was then diluted with additional THF and precipitated by addition to hexane. The solid was purified by centrifugation, and repeatedly dissolved in THF and reprecipitated with hexane until the supernatant was colorless. The solid was collected and dried.

Post-Polymerization Emulsification. A 0.05 wt \% solution of the aforementioned PEG-block-poly(1-alt-6)-block-PEG in THF was prepared and filtered with a $0.45 \mu \mathrm{m}$ nylon syringe filter. The THF solution $(0.1 \mathrm{~mL})$ was injected rapidly into $1.5 \mathrm{~mL}$ of Milli-Q water under vigorous stirring. The optically clear, pink dispersion was stirred open to air for $5 \mathrm{~min}$ and was then subjected to further analysis.

\section{RESULTS AND DISCUSSION}

Polymerization in Miniemulsion. In order to adjust the optical properties of polymer nanoparticles, different (co). monomers with variable electron accepting properties (Figure 1) were studied in the Sonogashira coupling to poly(arylene ethynyles). Dithiophene-substituted N,S-heterocycles were employed as these not only absorb at longer wavelengths, but in particular also possess a much higher solubility by comparison to the parent heterocycles. A stoichiometric amount of diethynyl bis(2-ethylhexyl)fluorene (1) was polymerized with mixtures of dibromo-substituted acceptor moieties (3 to 5) and dibromobis(2-ethylhexyl)fluorene (2). Incorporations of the electron-withdrawing repeat units were varied over a wide range, from $1 \mathrm{~mol} \%$ to $50 \mathrm{~mol} \%$ (relative to the total amount of monomers; that is, $50 \mathrm{~mol} \%$ corresponds to the acceptor monomer as the sole dibromo component).

Bromothiophene-substituted dihexylthienopyrazine 3 possesses the least pronounced electron withdrawing properties of the N,S-heterocycles investigated. Hexyl side chains were employed to provide sufficient solubility of the monomer in the organic phase of the miniemulsion protocol, as experiments with the methyl-substituted analogue revealed an insufficient solubility limiting the maximum incorporation of comonomer 3 to $4 \mathrm{~mol} \%$ (not shown here). This allowed for polymerization 
even with 3 as the sole dibromo component, to yield poly (1alt-3). Particle sizes are in the range of $40-60 \mathrm{~nm}$ (Table 1).

Table 1. Emulsion Polymerization with Different Portions of Dithiophene Thienopyrazine (Co)monomer $3^{a}$

\begin{tabular}{|c|c|c|c|c|c|}
\hline entry & (4mol] & $\begin{array}{l}3\left[\mathrm{~mol}_{0}\right) \\
(\mathrm{mol} \%)\end{array}$ & parficle size & $\begin{array}{l}\text { an, en } \\
\text { fim }\end{array}$ & {$[\%$} \\
\hline $1-1$ & 500 & $0(0 \%)$ & 47 & 498 & 26 \\
\hline $1-2$ & 490 & $10(1 \%)$ & 62 & 684 & 17 \\
\hline $1-3$ & 450 & $50(5 \%)$ & 49 & 704 & 6 \\
\hline $1-4$ & 400 & $100(10 \%)$ & 42 & 708 & 0.6 \\
\hline $1-5$ & 300 & $200(20 \%)$ & 57 & 713 & 0.5 \\
\hline $1-6$ & 0 & $500(50 \%)$ & 44 & 748 & 0.2 \\
\hline
\end{tabular}

aConditions: $0.500 \mathrm{mmol} \mathrm{1,2} \mathrm{wt} \mathrm{\%} \mathrm{(relative} \mathrm{to} \mathrm{water)} \mathrm{aqueous} \mathrm{SDS}$ solution (total volume $25 \mathrm{~mL}$ ) $1.0 \mathrm{~g}$ of toluene, $0.5 \mathrm{~g}$ of $i \mathrm{Pr}_{2} \mathrm{NH}_{4} 1$ $\mathrm{mol} \%$ (relative to the total amount of monomers) of $\left[\mathrm{Pd}\left(\mathrm{PPh}_{3}\right)_{4}\right]$, $100 \mu \mathrm{g}$ of Cul, $2 \mathrm{~min}$ ultrasonication, $72 \mathrm{~h}$ polymerization at $50^{\circ} \mathrm{C}$ ${ }^{b}$ Volume average particle size determined by DLS. "Excitation at 398 nm.

Molecular weights determined by GPC in THF solution vs polystyrene standards amount to $M_{\mathrm{n}}=5.0 \times 10^{4} ; M_{\mathrm{w}}=1.6 \times$ $10^{5}\left(M_{\mathrm{w}} / M_{\mathrm{n}} 3.1\right)$ for poly(1-alt-2) (entry $\left.1-1\right)$, and $M_{\mathrm{n}}=4.1 \times$ $10^{4} \mathrm{~g} \mathrm{~mol}^{-1} ; M_{\mathrm{w}}=1.5 \times 10^{5}\left(M_{\mathrm{w}} / M_{\mathrm{n}} 3.7\right)$ for poly [(1-alt-(2-co3)] with $1 \mathrm{~mol} \%$ of dithiophene(dihexyl)thienopyrazine repeat units (entry 1-2). Polymers with $5 \mathrm{~mol} \%$ or more of dithiophene(dihexyl)thienopyrazine repeat units possessed an insufficient solubility for GPC analysis, and poly(1-alt-3) (entry $1-6)$ is entirely insoluble in common organic solvents.

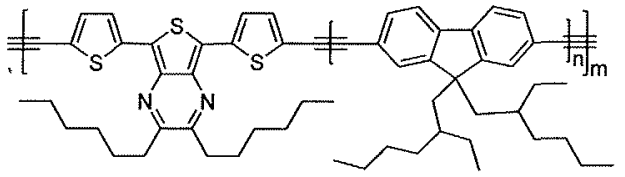

poly[1-alt-(2-co-3)] $(n=1$; poly $(1-a / t-3))$

The bis(benzothiadiazole) moiety is a stronger electron acceptor vs thienopyrazine, and provides enhanced absorption at higher wavelengths. The propensity for crystallization of the planar benzothiadiazole ring ${ }^{38-40}$ reduces the solubility of the monomer in the organic phase of the miniemulsion. In order to account for this, branched 2-ethylhexyl substituents were utilized on the thiophene rings (4). This enabled the polymerization even of 1 with 4 as the sole dibromo component even though 4-despite the branched side chains-has a limited solubility in the monomer/toluene phase of the miniemulsion. Consequently, the monomer and catalyst solution had to be processed rapidly to avoid recrystallization during emulsification. Stable dispersions were obtained for the entire range of $1 \mathrm{~mol} \%$ to $50 \mathrm{~mol} \%$ of 4 with particle sizes from $67-87 \mathrm{~nm}$ (Table S1, Supporting Information). Other than the polymers with di(thiophene)thienopyrazine repeat units, poly[1-alt-(2-co-4)] was largely insoluble in THF even at an incorporation of bis(benzothiadiazole) repeat units of only $1 \mathrm{~mol} \%$. A complete incorporation and absence of free monomer or other low molecular weight fractions was confirmed by thin layer chromatography (TLC) with a chloroform/petroleum ether mixture of a chloroform extract of poly (1-alt-4), along with a physical mixture of this polymer extract with monomer 4 , and the free monomer only for comparison. While the monomer was eluted from the latter two comparative samples, the polymer stayed in the origin entirely and no eluates were observed.

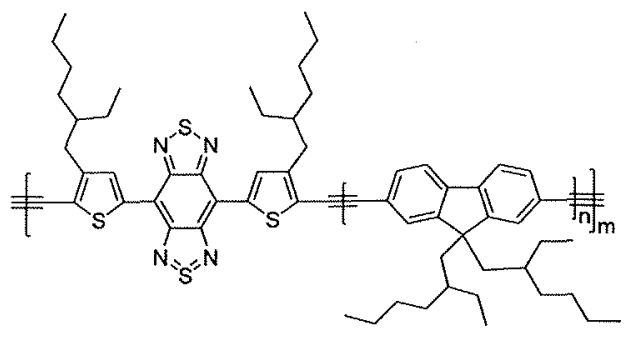

poly[1-alt-(2-co-4)] $(n=1$ : poly(1-alt-4))

In order to improve the solubility of the electron accepting monomer and to facilitate preparation and handling of the miniemulsion for polymerization, monomer 5 was studied (Table S2, Supporting Information). The annulated pyrazine vs thiodiazole ring and the additional aliphatic side chains were expected to reduce the electron withdrawing character slightly vs 4 , but the much improved solubility in toluene facilitated the handling in polymerizations of $\mathbf{5}$ in comparison to the aforementioned experiments. Stable dispersions of poly[1-alt(2-co-5)] with particle sizes around $70 \mathrm{~nm}$ were obtained, particles of poly(1-alt-5) (entry S2-5) were found to be larger. A quantitative incorporation of 5 could be underlined by TLC analogous to the analysis outlined above for poly(1-alt-4).

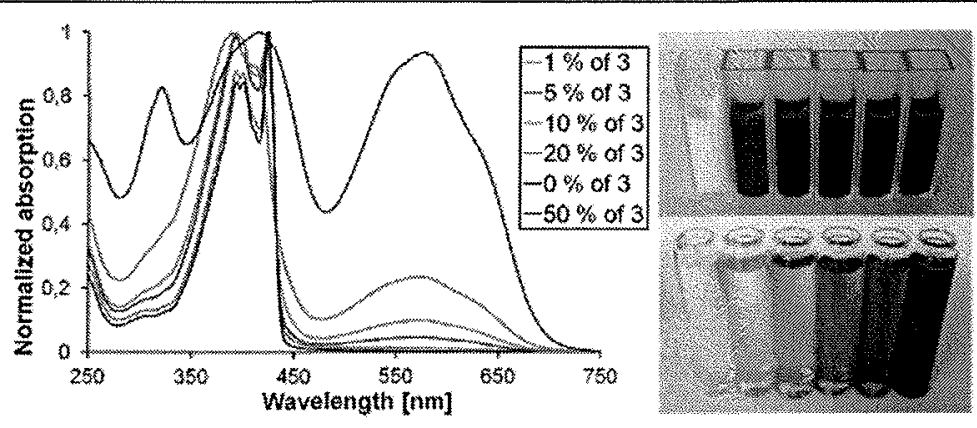

Figure 2. Absorption spectra of nanoparticles of poly [1-alt-(2-co-3)] with variable compositions, poly(1-alt-3), and poly(1-alt-2) for comparison (cf. Table 1). Top right: Photograph of the undiluted dispersions (left to right: 0 to $50 \mathrm{~mol} \% 3$ ). Bottom right: Dispersions diluted $10^{3}$-fold with water. 


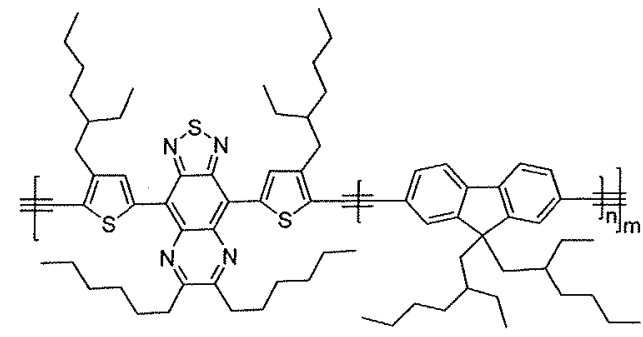

poly[1-alt-(2-co-5)] ( $n=1$ : poly(1-alt-5))

Optical Properties. Absorption and emission properties of the particle dispersions were studied in the UV-vis and NIR range, up to $1.4 \mu \mathrm{m}$ wavelength. As expected, in comparison to the all-fluorene based poly(fluorene ethynylene) (poly (1-alt2)), an increasingly intense absorption band at $575 \mathrm{~nm}$ is observed with increasing amounts of incorporated thienopyrazine repeat units in poly[1-alt-(2-co-3)] (Figure 2). This broad absorption over virtually the entire visible range accounts for the dark to black color of the dispersions even in a dilute state (Figure 2). Upon excitation with $\lambda=398 \mathrm{~nm}$, only a single emission band with a maximum at $\lambda_{\text {max,em }}=685-740 \mathrm{~nm}$, depending on the polymer composition, is observed (Table 1 and Figure 3 ). In a conjugated polymer nanoparticle, a large

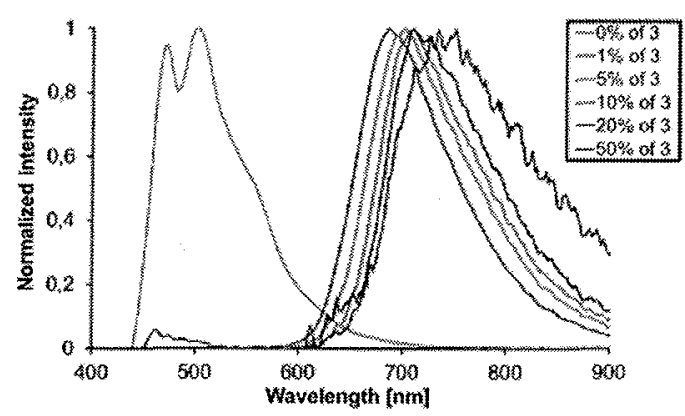

Figure 3. Fluorescence spectra of nanoparticles of poly[1-alt-(2-co-3)] with variable compositions, poly(1-alt-3), and poly(1-alt-2), for comparison (Table 1).

number of chromophores with different conjugation lengths and chemical compositions, and consequently energy levels exists. In the solid particle, intra- and interchain interactions result in energy transfer to the lower energy chromophores, which accounts for the larger Stokes shift vs polymer solutions. ${ }^{41,42}$ Before this well-established background, it is nonetheless notable that an incorporation of only $1 \mathrm{~mol} \%$ of monomer 3 suffices to trigger emission only from the lower energy states involving the heterocycle repeat units, virtually no fluorescence from the fluorene backbone was observed. Quantum yields decreased with increasing incorporations of 3 , which is probably due to self-quenching and reabsorption.

For poly[1-alt-(2-co-4)] dispersions, as anticipated absorption is extended to higher wavelengths in the form of a band with a maximum at $810-850 \mathrm{~nm}$, which increases in intensity with increasing amounts of incorporated 4 (Figure 4 and Table S1, Supporting Information). In the overall absorption spectrum, absorbance is low or virtually absent around 590 nm. This "spectral window" accounts for the greenish appearance of the dispersions (Figure 4). Other than for the dispersions of poly[1-alt-(2-co-3)] (vide supra), excitation at $398 \mathrm{~nm}$ did not result in any detectable fluorescence. However, an-albeit weak-NIR fluorescence at $900 \mathrm{~nm}$ was observed for polymer solutions in $\mathrm{CCl}_{4}$, excited with $\lambda=690 \mathrm{~nm}$ (Figure S4, Supporting Information). No fluorescence was found for the aqueous nanoparticle dispersions under these conditions. This is possibly due to absorption of the emitted fluorescence light by the dispersant water, as the solid state emission should be red-shifted in comparison to the aforementioned solution spectrum toward wavelengths where absorption by water is no longer negligible. ${ }^{43}$ Likely, a more pronounced nonradiative relaxation in the particles by comparison to polymers solutions also contributes.

As expected, the spectral properties of dispersions of poly [1alt-(2-co-5)] with the thiadiazoloquinoxaline motif are intermediate to those based on the bis(benzothiadiazole) and thienopyrazine moiety, respectively. Qualitatively, the absorption spectra are similar to poly[1-alt-(2-co-4)], with a significant blue shift of the lower energy band to $\lambda_{\max }=700$ (Figure S5, Supporting Information). Fluorescence could only be detected at a low incorporation of $1 \mathrm{~mol} \%$ of monomer 5 , with a low quantum yield of $\Phi=2 \%$ at $820 \mathrm{~nm}$.

Block Copolymer Synthesis. For the generation of selfstabilized conjugated polymer nanoparticles, an amphiphilic triblock copolymer with a central poly(arylene ethynylene) block and two terminal poly(ethylene glycol) blocks was prepared (Scheme 1). The conjugated block was prepared by Sonogoshira step growth polymerization in solution, with a slight deviation from stoichiometry in order to obtain alkyne end groups. Thorough removal of oxygen is essential to
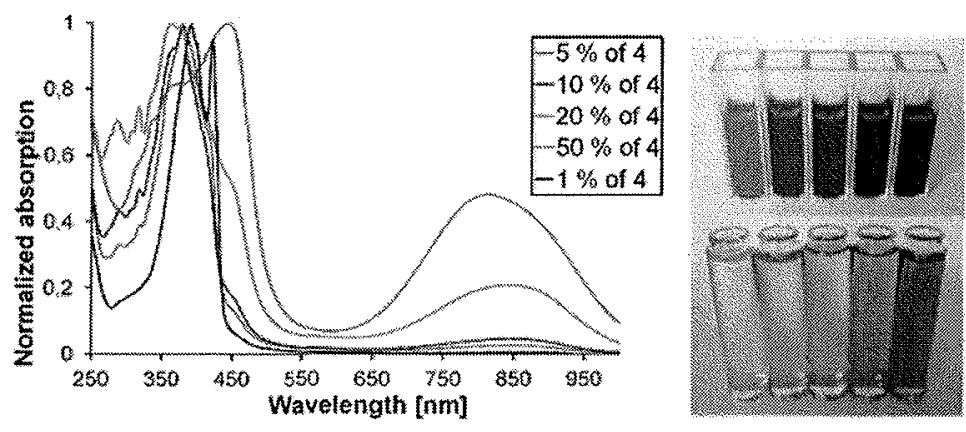

Figure 4. Absorption spectra of nanoparticles of poly[1-alt-(2-co-4)] with variable compositions, poly(1-alt-4), and poly(1-alt-2) for comparison (cf. Table S1, Supporting Information). Top right: Photograph of the undiluted dispersions (left to right: 0 to 50 mol \% 4). Bottom right: Dispersions diluted $10^{3}$-fold with water. 
Scheme 1. Synthetic Procedure for Amphiphilic Block Copolymer PEG-block-poly(1-alt-6)-block-PEG

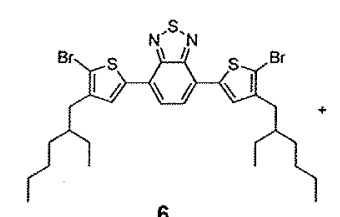

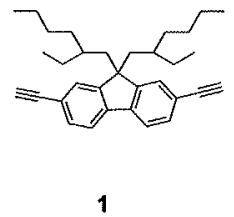

$\frac{\left[\mathrm{Pd}\left(\mathrm{PPh}_{3}\right)_{4}\right] / \mathrm{Cul}}{\text { toluene/piperidine }}$

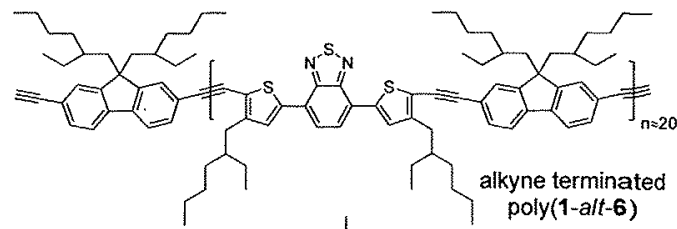

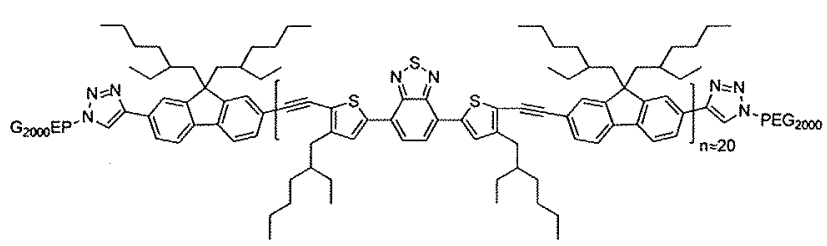

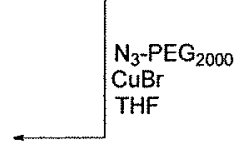

PEG-block-poly(1-alt-6)-block-PEG

suppress oxidative Glaser coupling as a side reaction. The formed alkyne-terminated poly(1-alt-6) was collected by precipitation in methanol. End group analysis by ${ }^{1} \mathrm{H}$ NMR spectroscopy reveals an average molecular weight of $M_{n}\left({ }^{1} \mathrm{H}\right.$ NMR $)=2.5 \times 10^{4} \mathrm{~g} \mathrm{~mol}^{-1}$, which corresponds to an average of 20 repeat units. GPC against polystyrene standards (Figure S7, Supporting Information) affords an apparent molecular weight of $M_{\mathrm{n}}=3.9 \times 10^{4} \mathrm{~g} \mathrm{~mol}^{-1}\left(M_{\mathrm{w}} / M_{\mathrm{n}} 2.5\right)$, which agrees reasonably with the absolute molecular weight from NMR determination taking into account that GPC will overestimate molecular weights of more rigid polymers. ${ }^{44}$ A THF solution of this poly (1-alt-6) was reacted with 2.1 equiv of polyethylene glycol monofunctionalized with an azide end group (PEGazide), with copper(I) bromide to promote the azide-alkyne addition reaction. Again, strict exclusion of oxygen was essential to suppress side reactions. After work-up, PEG-block-poly(1-alt6)-block-PEG was obtained as concluded from ${ }^{1} \mathrm{H}$ NMR analysis (Figure S3, Supporting Information). The characteristic resonance of the methylene protons ( $2.22 \mathrm{ppm}$ ) adjacent to the azide group is absent, indicating that no free unreacted PEG-azide is present. The absence of alkyne proton resonances $(3.12 \mathrm{ppm})$ agrees with complete functional group conversion. The PEG methylene protons adjacent to the triazole ring give rise to resonances at $4.62\left({ }^{\alpha} \mathrm{CH}_{2}\right)$ and 3.96 $\left({ }^{\beta} \mathrm{CH}_{2}\right)$ ppm, respectively. Note that the triazole proton resonance is obscured by the backbone signals.

Post-Polymerization Emulsification. Self-stabilized nanoparticles were generated by a rapid change of solvent quality ("nanoprecipitation") from a good solvent for both blocks to a dispersing medium that is miscible only with PEG. A dilute THF solution of the block copolymer was rapidly injected manually into a large excess of water. Particles with an average size of $55 \mathrm{~nm}$ according to DLS were formed. This particle size agrees with TEM analysis (Figure 5), which shows nonaggregated uniformly spherical particles. The dispersions are colloidally stable over prolonged times, as also evidenced by an unaltered particle size in repeated DLS measurements (Figure S8, Supporting Information).

The particle dispersion features a typical broad absorption, with maxima at 390 and $550 \mathrm{~nm}$ (Figure S6, Supporting Information). A single fluorescence band is observed with $\lambda_{\text {em max }}=672 \mathrm{~nm}$. An observed quantum yield of $\Phi=10 \%$ is satisfactory for a solid state polymer, making these particles well suited for spectral distinction from background fluorescence, for example in cell experiments.

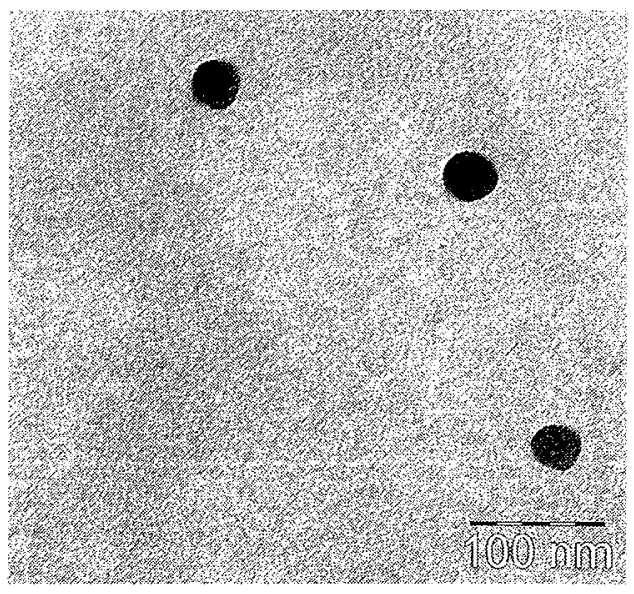

Figure 5. Electron microscopic image of nanoparticles of PEG-blockpoly (1-alt-6)-block-PEG.

\section{SUMMARY AND CONCLUSIONS}

Nanoparticles of conjugated polymers with an absorption profile in the red region of the spectrum and in the NIR regime up to $\lambda \approx 1 \mu \mathrm{m}$ can be generated in the form of poly(arylene ethynyles) containing electron-withdrawing annelated $S, N$ heterocycle motifs. Sonogashira coupling in aqueous miniemulsions of a diethynylene fluorene with appropriate dibromides afforded stable particle dispersion. With increasing content of the electron-withdrawing repeat units, the solubility of the polymers in organic solvents decreases strongly, and the materials are entirely insoluble in common solvents. This highlights the advantage of the miniemulsion procedure, which other than postpolymerization dispersion procedures can provide dispersions also of intractable, insoluble polymers. Note that a limited solubility even of the monomers in the organic phase of the miniemulsion, though much higher than the general solubility of the polymers, can be an issue for some of the compounds studied. For a poly(fluorene ethynylene) containing only $1 \mathrm{~mol} \%$ of di(thiophene)thienopyrazine repeat units, fluorescence was observed virtually exclusively in the red $\left(\lambda_{\text {em max }}\right.$ ca. $\left.700 \mathrm{~nm}\right)$. At the same time, the quantum yield of $\Phi$ $=17 \%$ is quite acceptable for a solid polymer. At higher incorporations, and for the more electron-withdrawing di(thiophene)-bis(benzothiadiazole) or -thiadiazoloquinoxaline 
repeat units fluorescence ceases. This is likely due to selfquenching and reabsorption.

For comparison, a postpolymerization approach by rapid change of solvent quality was applied to an amphiphilic blockcopolymer prepared for this purpose. Stable surfactantfree particles were generated from PEG-block-poly(1-alt-6)block-PEG (1, \{9,9-bis(2-ethylhexyl)-9H-fluoren-2,7-diyl\}ethynylene; 6, \{4,7-bis[4-(2-ethylhexyl)thiophene-2,5-diyl]benzothiadiazole ethynylene). These particles fluoresce in the orange-red regime, with $\lambda_{\mathrm{em} \max }$ ca. $680 \mathrm{~nm}$ and $\Phi=10 \%$. Note that this postpolymerization approach is restricted to the preparation of dispersions with considerably lower particle number concentrations vs the heterophase polymerization in miniemulsion polymerization as the approach requires utilization of dilute polymer solutions. The biocompatibility and nontoxicity of poly(ethylene glycol) ${ }^{45}$ is attractive when considering in vitro or in vivo imaging applications.

\section{AUTHOR INFORMATION}

\section{Corresponding Author}

*E-mail: Stefan.mecking@uni-konstanz.de.

\section{Author Contributions}

The manuscript was written through contributions of all authors. All authors have given approval to the final version of the manuscript.

Notes

The authors declare no competing financial interest.

\section{ACKNOWLEDGMENTS}

Financial support by the DFG (Me1388/7-1) is gratefully acknowledged. We thank Matthias Klein from the group of Andreas Zumbusch for NIR fluorescence measurements and access to UV-vis spectrometers. We also would like to thank Lars Bolk for GPC measurements and Arthur Groh for participation in some of this work as a part of his undergraduate studies.

\section{REFERENCES}

(1) Pecher, J.; Mecking, S. Chem. Rev. 2010, 110, 6260-6279.

(2) Landfester, $\mathrm{K}$; Montenegro, R; Scherf, U.; Guntner, R; Asawapirom, U.; Patil, S.; Neher, D.; Kietzke, T. Adv. Mater. 2002, $14,651-655$.

(3) Kietzke, T.; Neher, D.; Kumke, M.; Montenegro, R; Landfester, K; Scherf, U. Macromolecules 2004, 37, 4882-4890.

(4) Wu, C.; Szymanski, C.; Cain, Z.; McNeill, J. J. Am. Chem. Soc. 2007, 129, 12904-12905.

(5) Pecher, J.; Huber, J;; Winterhalder, M.; Zumbusch, A; Mecking, S. Biomacromolecules 2010, 11, 2776-2780.

(6) Rahim, N. A. A.; McDaniel, W.; Bardon, K; Srinivasan, S.; Vickermann, V.; So, P. T. C.; Moon, J. H. Adv. Mater. 2009, 21, 34923496.

(7) Wu, C; Bull, B.; Christensen, K.; McNeill, J. Angew. Chem., Int. Ed. 2009, 48, 2741-2745.

(8) Moon, J. H.; MacLean, P.; McDaniel, W.; Hancock, L. F. Chem. Commun. 2007, 4910-4912.

(9) Ye, F.; Wu, C.; Jin, Y.; Chan, Y.-H.; Zhang, X.; Chiu, D. T. J. Am Chem. Soc. 2011, 133, 8146-8149.
(10) Chong, H.; Nie, C.; Zhu, C.; Yang, Q.j Liu, L.; Lv, F.; Wang, S. Langmuir 2012, 28, 2091-2098.

(11) Huebner, C. F.; Foulger, S. H. Langmuir 2010, 26, 2945-2950.

(12) Zhuang, D.; Hogen-Esch, T. E. Macromolecules 2010, 43, 81708176.

(13) Andrew, T. L.; Swager, T. M. Macromolecules 2011, 44, 22762281 .

(14) Ye, F.; Wu, C.; Jin, Y.; Wang, M.; Chan, Y.-H.; Yu, J.; Sun, W.; Hayden, S.; Chiu, D. T. Chem. Commun. 2012, 48, 1778-1780.

(15) Cheng, L; Yang, K.; Chen, Q.; Liu, Z. ACS Nano 2012, 6, $5605-5613$.

(16) Jin, Y.; Ye, F.; Zeigler, M.; Wu, C.; Chiu, D. T. ACS Nano 2011, S, $1468-1475$

(17) Chan, Y.-H.; Ye, F.; Gallina, M. E.; Zhang, X.; Jin, Y.; Wu, I.-C.; Chiu, D. T. J. Am. Chem. Soc. 2012, 134, 7309-7312.

(18) Bundgaard, E.; Krebs, F. C. Sol. Energy Mater. Sol. Cells 2007, 91, 954-985.

(19) Winder, C.; Sariciftci, N. S. J. Mater. Chem. 2004, 14, 10771086.

(20) Pecher, J.; Mecking, S. Macromolecules 2007, 40, 7733-7735.

(21) Baier, M. C.; Huber, J.; Mecking, S. J. Am. Chem. Soc. 2009, 131, $14267-14273$

(22) Szymanski, C.; Wu, C.; Hooper, J.; Salazar, M. A.; Perdomo, A. Dukes, A.; McNeill, J. J. Phys. Chem. B 2005, 109, 8543-8546.

(23) Schütze, F,; Stempfle, B.; Jüngst, C.; Wöll, D.; Zumbusch, A.; Mecking, S. Chem. Commun. 2012, 48, 2104-2106.

(24) Kühne, A. J. C.; Weitz, D. A. Chem. Commun. 2011, 47, 1237912381 .

(25) Schmidt, M.; Amstub, R; Crass, G.; Seebach, D. Chem. Ber. $1980,113,1691-1707$

(26) Dai, C.; Fu, C. F. J. Am. Chem. Soc. 2001, 123, 2719-2724.

(27) Jo, J.; Chi, C.; Höger, S.; Wegner, G.; Yoon, D. Y. Chem--Eur. J. 2004, 10, 2681-2688.

(28) Mozingo, R; Harris, S. A.; Wolf, D. E.; Hoffhine, C. E., Jr.; Easton, N. R; Folkers, K. J. Am. Chem. Soc. 1945, 67, 2092-2095.

(29) Xia, Y.; Luo, J.; Deng, X.; Li, X.; Li, D.; Zhu, X.; Yang, W.; Cao, Y. Macromol. Chem. Phys. 2006, 207, 511-520.

(30) Kitamura, C.; Tanaka, S.; Yamashita, Y. Chem. Mater. 1996, 8 $570-578$.

(31) He, Y.; Wang, X.; Zhang, J.; Li, Y. Macromol. Rapid Commun 2009, 30, 45-51.

(32) Toshio, U.; Kaname, T.; Munemitsu, T. Chem. Pharm. Bull 1980, 28, 1909-1912.

(33) Karikomi, M.; Kitamura, C.; Tanaka, S.; Yamashita, Y. J. Am. Chem. Soc. 1995, $117,6791-6792$.

(34) Ram, S.; Ehrenkaufer, R. E. Tetrahedron Lett. 1984, 25, 34153418

(35) Hong, D.J.; Lee, E.; Jeong, H.; Lee, J.-k.; Zin, W.-C.; Nguyen, T. D.; Glotzer, S. C.; Lee, M. Angew. Chem., Int. Ed. 2009, 48, 16641668

(36) Hoffmann, R. W.; Metternich, R.; Lanz, J. W. Liebigs Ann. Chem. $1987,10,881-887$

(37) Gautrot, J. E.; Hodge, P.; Cupertino, D.; Helliwell, M. New I. Chem. 2007, 31, 1585-1593.

(38) Suzuki, T.; Yamashita, Y.; Fukushima, T.; Miyashi, T. Mol. Cryst. Liq. Cryst. 1997, 296, 165-180.

(39) Akhtaruzzaman, Md.; Tomura, M.; Zaman, Md. B.; Nishida, J.; Yamashita, Y. J. Org. Chem. 2002, 67, 7813-7818.

(40) Ono, K; Tanaka, S.; Yamashita, Y. Angew. Chem. 1994, 33, 1977-1979.

(41) Schwartz, B. J. Annu. Rev. Phys. Chem. 2003, 54, 141-172.

(42) Grey, J. K.; Kim, D. Y.; Norris, B. C.; Miller, W. L.; Barbara, P. F. J. Phys. Chem. B 2006, 110, 25568-25572.

(43) Matcher, S. J.; Cope, M.; Delpy, D. T. Phys. Med. Biol. 1993, 38 , $177-196$

(44) Grell, M.; Bradley, D. D. C.; Long, X; Chamberlain, T.; Inbasekaran, M.; Woo, E. P.; Soliman, M. Acta Polym. 1998, 49, 439444. 
(45) Katz, L. C.; Burkhalter, A.; Dreyer, W. J. Nature 1984, 310, 498-500. 